\title{
Studies on Effect of Different Packaging Materials and Storage Temperature on Physiological Loss in Weight of Amaranthus (Amaranthus viridis)
}

\author{
M. S. Marichamy*, J. Jyothsna, S. Harini, V. Bhuvaneswari Devi, \\ B. Rajapriya, A. Rajalakshmi and R. Ahaljith
}
Department of Horticulture, Pandit Jawaharlal Nehru College of Agriculture and Research Institute, Karaikal, U.T. of Puducherry, India

*Corresponding author

\section{A B S T R A C T}

\begin{tabular}{|l|}
\hline Key w o r d s \\
Amaranthus, PLW, \\
Packaging \\
materials, Plastic \\
Crates, ZECC, \\
Ambient storage \\
\hline Article Info \\
\hline $\begin{array}{l}\text { Accepted: } \\
\text { 07 September } 2020 \\
\text { Available Online: } \\
\text { 10 October } 2020\end{array}$ \\
\hline
\end{tabular}

The experiment was conducted in the Department of Horticulture, Pandit Jawaharlal Nehru College of Agriculture and Research Institute, Karaikal from January 2016 to March 2016 to standardize the effect of different packaging materials and storage temperature on physiological loss in weight of amaranths. The experiment was laid out in CRD with ten treatments and three replications. The present study, $100 \mathrm{~g}$ of freshly harvested, tender green leaves along with roots were weighed and packed in different packing materials viz., plastic crates, 200 and 300 gauge polyethylene bag without and 1 per cent perforation followed by stored under ambient storage $\left(25^{\circ} \mathrm{C} \pm 2^{\circ} \mathrm{C}\right.$ and $\left.50.33-73.66 \% \mathrm{RH}\right)$ and Zero Energy Cool Chamber at $22.36-24.73^{\circ} \mathrm{C}$ and $92.66-97.36 \% \mathrm{RH}$. The physiological loss in weight was recorded at four hours interval until loss of freshness. Generally, PLW progressively increased with storage period from 4 hours after storage to 96 hours after storage with greater increases in amaranthus packed in plastic crates and stored under ambient condition $\left(\mathrm{T}_{10}\right)$. Among the different treatments, the treatment $\mathrm{T}_{1}$ (Amaranthus packed in 200 gauge polyethylene bag without perforation and stored under ZECC) recorded the lowest PLW compared to other treatments. It was followed by $\left(\mathrm{T}_{2}\right)$ Amaranthus packed in 200 gauge polyethylene bag with 1 per cent perforation and stored under ZECC).

\section{Introduction}

Amaranthus is the most important leafy vegetables of South India mostly cultivated in Kerala, Tamil Nadu, Karnataka, Maharashtra, Andhra Pradesh, Telangana. Amaranthus is primarily used as a potherb. It is the most common leafy vegetable grown during summer and rainy season in India. The fresh tender leaves and stem give delicious preparation on cooking. It belongs to the family Amaranthaceae. Amaranthus is considered to be the cheapest leafy vegetables in the market and it could be rightly described as a "poor man's vegetable".

Amaranth is an excellent source of calcium, magnesium, potassium, vitamin $\mathrm{C}$, vitamin 
B6, folate, vitamin $\mathrm{A}$, and an incredible source of vitamin K. Amaranth leaves, rich in calcium and beta-carotene, keep bones strong and reduces one's risk of osteoporosis.

The post-harvest loss in amaranthus occurs due to improper harvesting, lack of proper packaging materials, improper handling during long-distance transport, lack of storage, and microbial spoilage. The postharvest losses may be reduced by adopting necessary cultural operations, careful handling, and packing, and also by different storage methods such as refrigeration, controlled atmospheric storage, pre-packing, ZECC, etc. Among these methods, ZECC and packaging are most suitable for slowing down the biological activity of the produce, growth, and spread of microorganisms and to reduce wilting and decaying. Low temperature can slow down the respiration considerably and prolong the storage life.

Though amaranthus is nutritious and fetches good returns, it suffers from a very poor shelf life because of the high amount of moisture content in the leaves. The postharvest shelf life of amaranthus was one day at ambient room temperature. Therefore, there is a need to prolong the shelf life of the amaranthus for at least one week. Hence, the present investigation was carried out to know the effect of packaging materials and storage temperature on reducing the physiological loss in weight of amaranthus.

\section{Materials and Methods}

The experiments were conducted at the Department of Horticulture, Pandit Jawaharlal Nehru College of Agriculture and Research Institute, Karaikal from January 2016 to March 2016. The amaranthus was raised under irrigated conditions during January 2016 by adopting the recommended package of practices. Twenty-five days after sowing the crop was ready for harvesting. Uniform, tender mature fresh greens were harvested along with stem during morning hours. They were immediately brought to the laboratory then washed and surface dried leaves and used for this experiment. The experiment was laid out in a Completely Randomized Design (CRD) with ten treatments and replicated thrice. The treatments comprising of viz., $\mathrm{T}_{1}$ : Amaranthus packed in 200 gauge polyethylene bag without perforation and stored under ZECC; $\mathrm{T}_{2}$ : Amaranthus packed in 200 gauge polyethylene bag with 1 per cent perforation and stored under ZECC; $\mathrm{T}_{3}$ : Amaranthus packed in 300 gauge polyethylene bag without perforation and stored under ZECC; $\mathrm{T}_{4}$ : Amaranthus packed in 300 gauge polyethylene bag with 1 per cent perforation and stored under ZECC; $\mathrm{T}_{5}$ : Amaranthus packed in plastic crates and stored under ZECC; $\mathrm{T}_{6}$ : Amaranthus packed in 200 gauge polyethylene bag without perforation and stored under ambient condition; $\mathrm{T}_{7}$ : Amaranthus packed in 200 gauge polyethylene bag with 1 per cent perforation and stored under ambient condition; $\mathrm{T}_{8}$ : Amaranthus packed in 300 gauge polyethylene bag without perforation and stored under ambient condition; $\mathrm{T}_{9}$ : Amaranthus packed in 300 gauge polyethylene bag with 1 per cent perforation and stored under ambient condition; $\mathrm{T}_{10}$ : Amaranthus packed in plastic crates and stored under ambient condition.

The size of the polyethylene bag used was $45 \times 30 \mathrm{~cm}$. Perforation was provided by punching holes on both the sides of the bag to the extent of 1 per cent perforation of the total surface area. In the present study, $100 \mathrm{~g}$ of freshly harvested, tender green leaves along with roots were weighed and packed in different packing materials viz., plastic crates, 200 and 300 gauge without and 1 per cent perforation followed by ambient storage $\left(25^{\circ} \mathrm{C} \pm 2{ }^{\circ} \mathrm{C}\right.$ and $\left.50.33-73.66 \% \mathrm{RH}\right)$ and 
Zero Energy Cool Chamber at 22.36 $24.73^{\circ} \mathrm{C}$ and $92.66-97.36 \% \mathrm{RH}$.

The shelf life of the stored samples was studied by recording the physiological loss in weight (PLW \%) throughout the study period for all the samples every four hours intervals. The physiological loss in weight (PLW) was calculated by using the following formula and the mean was expressed in percentage (Sankaran, 1999).

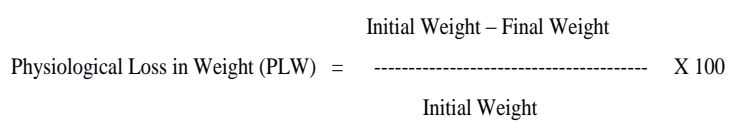

\section{Results and Discussion}

Effect of different packaging materials and storage temperature on physiological loss in weight of amaranthus

Generally, physiological loss in weight progressively increased with storage period from 4 hours after storage (HAS) to 96 hours after storage (HAS) with greater increases in amaranthus packed in plastic crates and stored under ambient condition $\left(T_{1}\right)$. After 96 hours after storage, the physiological loss in weight of amaranthus ranged from 0.00 per cent to 66.0 percent. (Table.1a)

At the end of the 24 HAS $\left(1^{\text {st }}\right.$ day), the physiological loss in weight was almost nil because only 0.2 per cent was recorded in amaranthus packed in 200 gauge polyethylene bag without perforation and stored under ZECC $\left(T_{1}\right)$. It was closely followed by the treatments $\mathrm{T}_{2},(0.40$ per cent $) \mathrm{T}_{6}(0.40$ per cent $)$ and $\mathrm{T}_{3}(0.8$ per cent $)$. It was also pertinent to note that the treatments $\mathrm{T}_{4}, \mathrm{~T}_{7}$, and $\mathrm{T}_{8}$ were on par with each other. While the treatment $\mathrm{T}_{10}$ recorded the highest physiological loss in weight of 25 percent and followed by the treatment $\left(T_{5}\right)$ which recorded 9.0 percent (Table 1a).
Till the end of the 48 HAS ( $2^{\text {nd }}$ day) the physiological loss in weight in amaranthus only 1 percent was recorded in amaranthus packed in 200 gauge polyethylene bag without perforation and stored under ZECC $\left(\mathrm{T}_{1}\right)$. The treatments $\mathrm{T}_{2}$ and $\mathrm{T}_{6}$ (1.1 per cent) were on par with each other. The treatment $\left(\mathrm{T}_{10}\right) \quad$ which recorded the highest physiological loss in weight of 43 percent and followed by the treatment $\left(T_{5}\right)$ recorded 16.0 percent (Table 1b).

Till the end of the 72 HAS ( $3^{\text {rd }}$ day) the physiological loss in weight in amaranthus only 2 percent was recorded in amaranthus packed in 200 gauge polyethylene bag without perforation and stored under ZECC $\left(T_{1}\right)$. It was followed by treatments $T_{2}$ and $T_{6}$ which recorded 2.5 per cent of physiological loss in weight. It was also observed that treatments $T_{3}\left(2.6\right.$ per cent) $T_{4}$ and $T_{7}$ (3.0 per cent) were on par with each other. The treatment $\left(\mathrm{T}_{10}\right)$ which recorded the highest physiological loss in weight of 55 percent and followed by the treatment $\left(\mathrm{T}_{5}\right)$ recorded 27.5 percent. (Table.1c)

At the end of the 96 HAS ( $4^{\text {th }}$ day), the physiological loss in weight was 4.0 per cent was recorded in amaranthus packed in 200 gauge polyethylene bag without perforation and stored under ZECC $\left(\mathrm{T}_{1}\right)$. It was closely followed by the treatments $\mathrm{T}_{2}$, (4.5 per cent) $\mathrm{T}_{6}$ (4.7 per cent) and $\mathrm{T}_{3}$ ( 4.9 per cent). While the treatment $\mathrm{T}_{10}$ recorded the highest physiological loss in weight of 66 percent and followed by the treatment $\left(T_{5}\right)$ which recorded 37.0 percent (Table 1d).

It is evident from Table 1e. The physiological loss in weight ranged from 24 HAS, 48 HAS, 72 HAS and 96 HAS where the variation was high within the hours. The first two days (24 HAS and 48 HAS) the loss in PLW there was not much effect in the significant differences. 
Table.1 Effect of different packaging materials and storage temperature on Physiological loss in weight (per cent) of amaranthus - 4 to 96 has

\begin{tabular}{|c|c|c|c|c|c|c|c|c|c|c|c|c|}
\hline \multirow[t]{2}{*}{ Treatments } & \multicolumn{6}{|c|}{$1^{\text {st }}$ Day After Storage (DAS) } & \multicolumn{6}{|c|}{$2^{\text {nd }}$ Day After Storage (DAS) } \\
\hline & 4 HAS & 8 HAS & 12 HAS & 16 HAS & 20 HAS & 24 HAS & 28 HAS & 32 HAS & 36 HAS & 40 HAS & 44 HAS & 48 HAS \\
\hline $\mathbf{T}_{1}$ & 0.00 & 0.10 & 0.10 & 0.10 & 0.10 & 0.20 & 0.30 & 0.50 & 0.50 & 0.50 & 0.50 & 1.00 \\
\hline $\mathbf{T}_{2}$ & 0.10 & 0.20 & 0.20 & 0.20 & 0.20 & 0.40 & 0.50 & 0.80 & 0.80 & 0.80 & 0.80 & 1.10 \\
\hline $\mathbf{T}_{\mathbf{3}}$ & 0.70 & 0.70 & 0.70 & 0.70 & 0.80 & 0.80 & 1.00 & 1.00 & 1.20 & 1.20 & 1.20 & 1.20 \\
\hline $\mathbf{T}_{4}$ & 1.10 & 1.20 & 1.20 & 1.20 & 1.20 & 1.50 & 1.70 & 1.70 & 1.80 & 1.80 & 2.00 & 2.10 \\
\hline $\mathbf{T}_{5}$ & 3.50 & 5.50 & 7.00 & 8.00 & 8.50 & 9.00 & 10.50 & 11.50 & 12.00 & 13.50 & 14.50 & 16.00 \\
\hline $\mathbf{T}_{6}$ & 0.30 & 0.40 & 0.40 & 0.40 & 0.40 & 0.40 & 0.50 & 0.90 & 1.00 & 1.00 & 1.00 & 1.10 \\
\hline $\mathbf{T}_{7}$ & 1.00 & 1.00 & 1.10 & 1.20 & 1.30 & 1.40 & 1.50 & 1.50 & 1.70 & 1.90 & 1.90 & 1.90 \\
\hline $\mathbf{T}_{8}$ & 1.60 & 1.60 & 1.60 & 1.60 & 1.60 & 1.70 & 2.00 & 2.00 & 2.00 & 2.00 & 2.00 & 2.10 \\
\hline $\mathbf{T}_{9}$ & 1.90 & 2.00 & 2.10 & 2.10 & 2.20 & 2.20 & 2.50 & 3.00 & 3.00 & 3.00 & 3.00 & 3.20 \\
\hline $\mathbf{T}_{10}$ & 6.00 & 10.50 & 15.50 & 18.50 & 21.50 & 25.00 & 29.00 & 31.50 & 36.00 & 38.50 & 42.00 & 43.00 \\
\hline SEd & 0.4260 & 0.6901 & 0.9743 & 1.1489 & 1.3148 & 1.5089 & 1.7510 & 1.9049 & 2.1520 & 2.3122 & 2.5156 & 2.5987 \\
\hline CD & $\begin{array}{c}0.8886 * \\
*\end{array}$ & $\begin{array}{c}1.4395 * \\
*\end{array}$ & $2.0324 * *$ & $\begin{array}{c}2.3965 * \\
*\end{array}$ & $\begin{array}{c}2.7426 * \\
*\end{array}$ & $\begin{array}{c}3.1474 * \\
*\end{array}$ & $\begin{array}{c}3.6526 * \\
*\end{array}$ & $\begin{array}{c}3.9735 * \\
*\end{array}$ & $\begin{array}{c}4.4890 * \\
*\end{array}$ & $4.8231 * *$ & $5.2474 * *$ & $\begin{array}{c}5.4208 * \\
*\end{array}$ \\
\hline
\end{tabular}

Treatment details

\begin{tabular}{|c|c|}
\hline $\mathbf{T}_{\mathbf{1}}$ & Amaranthus packed in 200 gauge polyethylene bag without perforation and stored under ZECC \\
\hline $\mathbf{T}_{\mathbf{2}}$ & Amaranthus packed in 200 gauge polyethylene bag with 1 per cent perforation and stored under ZECC. \\
\hline $\mathbf{T}_{\mathbf{3}}$ & Amaranthus packed in 300 gauge polyethylene bag without perforation and stored under ZECC. \\
\hline $\mathbf{T}_{\mathbf{4}}$ & Amaranthus packed in 300 gauge polyethylene bag with 1 per cent perforation and stored under ZECC. \\
\hline $\mathbf{T}_{\mathbf{5}}$ & Amaranthus packed in plastic crates and stored under ZECC. \\
\hline $\mathbf{T}_{\mathbf{6}}$ & Amaranthus packed in 200 gauge polyethylene bag without perforation and stored under ambient condition \\
\hline $\mathbf{T}_{\mathbf{7}}$ & Amaranthus packed in 200 gauge polyethylene bag with 1 per cent perforation and stored under ambient condition \\
\hline $\mathbf{T}_{\mathbf{8}}$ & Amaranthus packed in 300 gauge polyethylene bag without perforation and stored under ambient condition \\
\hline $\mathbf{T}_{\mathbf{9}}$ & Amaranthus packed in 300 gauge polyethylene bag with 1 per cent perforation and stored under ambient condition \\
\hline $\mathbf{T}_{\mathbf{1 0}}$ & Amaranthus packed in plastic crates and stored under ambient condition. \\
\hline
\end{tabular}


Table.1 Cont

\begin{tabular}{|c|c|c|c|c|c|c|c|c|c|c|c|c|}
\hline \multirow{2}{*}{$\begin{array}{l}\text { Treatment } \\
\text { s }\end{array}$} & \multicolumn{6}{|c|}{$3^{\text {rd }}$ Day After Storage (DAS) } & \multicolumn{6}{|c|}{$4^{\text {th }}$ Day After Storage (DAS) } \\
\hline & 52 HAS & 56 HAS & 60 HAS & 64 HAS & 68 HAS & 72 HAS & 76 HAS & 80 HAS & 84 HAS & 88 HAS & 92 HAS & 96 HAS \\
\hline $\mathrm{T}_{1}$ & 1.10 & 1.20 & 1.30 & 1.70 & 1.80 & 2.00 & 2.30 & 2.50 & 2.80 & 3.00 & 3.50 & 4.00 \\
\hline $\mathbf{T}_{2}$ & 1.20 & 1.30 & 1.50 & 1.80 & 1.90 & 2.50 & 2.70 & 2.80 & 3.00 & 3.50 & 4.00 & 4.50 \\
\hline $\mathbf{T}_{3}$ & 1.30 & 1.40 & 1.60 & 2.00 & 2.20 & 2.60 & 3.00 & 3.00 & 3.20 & 3.70 & 4.50 & 4.90 \\
\hline $\mathbf{T}_{4}$ & 2.10 & 2.10 & 2.30 & 2.50 & 2.50 & 3.00 & 3.30 & 3.60 & 3.70 & 4.20 & 4.70 & 5.20 \\
\hline $\mathbf{T}_{5}$ & 17.50 & 19.00 & 20.50 & 22.00 & 26.50 & 27.50 & 28.50 & 29.50 & 31.50 & 32.50 & 35.00 & 37.00 \\
\hline $\mathbf{T}_{6}$ & 1.20 & 1.40 & 1.60 & 2.00 & 2.00 & 2.50 & 2.80 & 2.90 & 3.00 & 3.60 & 4.20 & 4.70 \\
\hline $\mathbf{T}_{7}$ & 2.00 & 2.00 & 2.20 & 2.40 & 2.50 & 3.00 & 3.10 & 3.50 & 3.50 & 4.00 & 4.50 & 5.00 \\
\hline $\mathbf{T}_{8}$ & 2.10 & 2.30 & 2.50 & 3.00 & 3.00 & 3.50 & 3.80 & 3.90 & 4.00 & 4.50 & 5.00 & 5.50 \\
\hline $\mathbf{T}_{9}$ & 3.20 & 3.30 & 3.50 & 3.60 & 3.70 & 4.00 & 4.20 & 4.50 & 4.70 & 5.00 & 5.50 & 6.00 \\
\hline $\mathbf{T}_{10}$ & 45.50 & 47.50 & 48.00 & 49.50 & 52.50 & 55.00 & 57.00 & 59.00 & 61.00 & 62.00 & 64.00 & 66.00 \\
\hline SEd & 2.7599 & 2.8962 & 2.9574 & 3.0734 & 3.3336 & 3.4122 & 3.6222 & 3.7511 & 3.9041 & 3.9899 & 4.1675 & 4.3318 \\
\hline CD & $\begin{array}{c}\text { 5.7570* } \\
*\end{array}$ & $6.0414 * *$ & $6.1690 * *$ & 6.4111** & $6.9538 * *$ & $7.2846 * *$ & $7.5558 * *$ & $7.8247 * *$ & $8.1439 * *$ & $\begin{array}{c}8.3227 * \\
*\end{array}$ & $8.6933 * *$ & $9.0360 * *$ \\
\hline
\end{tabular}

Treatment details

\begin{tabular}{|c|c|}
\hline $\mathbf{T}_{\mathbf{1}}$ & Amaranthus packed in $\mathbf{2 0 0}$ gauge polyethylene bag without perforation and stored under ZECC \\
\hline $\mathbf{T}_{\mathbf{2}}$ & Amaranthus packed in 200 gauge polyethylene bag with 1 per cent perforation and stored under ZECC. \\
\hline $\mathbf{T}_{\mathbf{3}}$ & Amaranthus packed in 300 gauge polyethylene bag without perforation and stored under ZECC. \\
\hline $\mathbf{T}_{\mathbf{4}}$ & Amaranthus packed in 300 gauge polyethylene bag with 1 per cent perforation and stored under ZECC. \\
\hline $\mathbf{T}_{\mathbf{5}}$ & Amaranthus packed in plastic crates and stored under ZECC. \\
\hline $\mathbf{T}_{\mathbf{6}}$ & Amaranthus packed in 200 gauge polyethylene bag without perforation and stored under ambient condition \\
\hline $\mathbf{T}_{\mathbf{7}}$ & Amaranthus packed in 200 gauge polyethylene bag with 1 per cent perforation and stored under ambient condition \\
\hline $\mathbf{T}_{\mathbf{8}}$ & Amaranthus packed in 300 gauge polyethylene bag without perforation and stored under ambient condition \\
\hline $\mathbf{T}_{\mathbf{9}}$ & Amaranthus packed in 300 gauge polyethylene bag with 1 per cent perforation and stored under ambient condition \\
\hline $\mathbf{T}_{\mathbf{1 0}}$ & Amaranthus packed in plastic crates and stored under ambient condition. \\
\hline
\end{tabular}


Table.1a Effect of different packaging materials and storage temperature on physiological loss in weight (per cent) of amaranthus - 4 to 24 has $\left(1^{\text {st }}\right.$ day after storage)

\begin{tabular}{|c|c|c|c|c|c|c|}
\hline \multirow[t]{2}{*}{ Treatments } & \multicolumn{6}{|c|}{$1^{\text {st }}$ Day after storage (DAS) } \\
\hline & 4 HAS & 8 HAS & 12 HAS & 16 HAS & 20 HAS & 24 HAS \\
\hline $\mathbf{T}_{1}$ & 0.00 & 0.10 & 0.10 & 0.10 & 0.10 & 0.20 \\
\hline $\mathbf{T}_{2}$ & 0.10 & 0.20 & 0.20 & 0.20 & 0.20 & 0.40 \\
\hline $\mathbf{T}_{3}$ & 0.70 & 0.70 & 0.70 & 0.70 & 0.80 & 0.80 \\
\hline $\mathbf{T}_{4}$ & 1.10 & 1.20 & 1.20 & 1.20 & 1.20 & 1.50 \\
\hline $\mathbf{T}_{5}$ & 3.50 & 5.50 & 7.00 & 8.00 & 8.50 & 9.00 \\
\hline $\mathbf{T}_{6}$ & 0.30 & 0.40 & 0.40 & 0.40 & 0.40 & 0.40 \\
\hline $\mathbf{T}_{7}$ & 1.00 & 1.00 & 1.10 & 1.20 & 1.30 & 1.40 \\
\hline $\mathbf{T}_{8}$ & 1.60 & 1.60 & 1.60 & 1.60 & 1.60 & 1.70 \\
\hline $\mathbf{T}_{9}$ & 1.90 & 2.00 & 2.10 & 2.10 & 2.20 & 2.20 \\
\hline $\mathbf{T}_{10}$ & 6.00 & 10.50 & 15.50 & 18.50 & 21.50 & 25.00 \\
\hline SEd & 0.4260 & 0.6901 & 0.9743 & 1.1489 & 1.3148 & 1.5089 \\
\hline CD (0.05) & $0.8886 * *$ & $1.4395 * *$ & $2.0324 * *$ & $2.3965 * *$ & $2.7426 * *$ & $3.1474 * *$ \\
\hline
\end{tabular}

Treatment details

\begin{tabular}{|c|c|}
\hline $\mathbf{T}_{\mathbf{1}}$ & Amaranthus packed in $\mathbf{2 0 0}$ gauge polyethylene bag without perforation and stored under ZECC \\
\hline $\mathbf{T}_{\mathbf{2}}$ & Amaranthus packed in 200 gauge polyethylene bag with 1 per cent perforation and stored under ZECC. \\
\hline $\mathbf{T}_{\mathbf{3}}$ & Amaranthus packed in 300 gauge polyethylene bag without perforation and stored under ZECC. \\
\hline $\mathbf{T}_{\mathbf{4}}$ & Amaranthus packed in 300 gauge polyethylene bag with 1 per cent perforation and stored under ZECC. \\
\hline $\mathbf{T}_{\mathbf{5}}$ & Amaranthus packed in plastic crates and stored under ZECC. \\
\hline $\mathbf{T}_{\mathbf{6}}$ & Amaranthus packed in 200 gauge polyethylene bag without perforation and stored under ambient condition \\
\hline $\mathbf{T}_{\mathbf{7}}$ & Amaranthus packed in 200 gauge polyethylene bag with 1 per cent perforation and stored under ambient condition \\
\hline $\mathbf{T}_{\mathbf{8}}$ & Amaranthus packed in 300 gauge polyethylene bag without perforation and stored under ambient condition \\
\hline $\mathbf{T}_{\mathbf{9}}$ & Amaranthus packed in 300 gauge polyethylene bag with 1 per cent perforation and stored under ambient condition \\
\hline $\mathbf{T}_{\mathbf{1 0}}$ & Amaranthus packed in plastic crates and stored under ambient condition. \\
\hline
\end{tabular}


Table.1b Effect of different packaging materials and storage temperature on physiological loss in weight (per cent) of amaranthus - 28 to 48 has $\left(2^{\text {nd }}\right.$ day after storage)

\begin{tabular}{|c|c|c|c|c|c|c|}
\hline \multirow[t]{2}{*}{ Treatments } & \multicolumn{6}{|c|}{$2^{\text {nd }}$ Day after storage (DAS) } \\
\hline & 28 HAS & 32 HAS & 36 HAS & 40 HAS & 44 HAS & 48 HAS \\
\hline $\mathbf{T}_{1}$ & 0.30 & 0.50 & 0.50 & 0.50 & 0.50 & 1.00 \\
\hline $\mathbf{T}_{2}$ & 0.50 & 0.80 & 0.80 & 0.80 & 0.80 & 1.10 \\
\hline $\mathbf{T}_{\mathbf{3}}$ & 1.00 & 1.00 & 1.20 & 1.20 & 1.20 & 1.20 \\
\hline $\mathbf{T}_{4}$ & 1.70 & 1.70 & 1.80 & 1.80 & 2.00 & 2.10 \\
\hline $\mathbf{T}_{5}$ & 10.50 & 11.50 & 12.00 & 13.50 & 14.50 & 16.00 \\
\hline $\mathbf{T}_{6}$ & 0.50 & 0.90 & 1.00 & 1.00 & 1.00 & 1.10 \\
\hline $\mathbf{T}_{7}$ & 1.50 & 1.50 & 1.70 & 1.90 & 1.90 & 1.90 \\
\hline $\mathbf{T}_{8}$ & 2.00 & 2.00 & 2.00 & 2.00 & 2.00 & 2.10 \\
\hline $\mathbf{T}_{9}$ & 2.50 & 3.00 & 3.00 & 3.00 & 3.00 & 3.20 \\
\hline $\mathbf{T}_{10}$ & 29.00 & 31.50 & 36.00 & 38.50 & 42.00 & 43.00 \\
\hline SEd & 1.7510 & 1.9049 & 2.1520 & 2.3122 & 2.5156 & 2.5987 \\
\hline CD (0.05) & $3.6526 * *$ & $3.9735 * *$ & $4.4890 * *$ & $4.8231 * *$ & $5.2474 * *$ & $5.4208 * *$ \\
\hline
\end{tabular}

Treatment details

\begin{tabular}{|c|c|}
\hline $\mathbf{T}_{\mathbf{1}}$ & Amaranthus packed in $\mathbf{2 0 0}$ gauge polyethylene bag without perforation and stored under ZECC \\
\hline $\mathbf{T}_{\mathbf{2}}$ & Amaranthus packed in 200 gauge polyethylene bag with 1 per cent perforation and stored under ZECC. \\
\hline $\mathbf{T}_{\mathbf{3}}$ & Amaranthus packed in 300 gauge polyethylene bag without perforation and stored under ZECC. \\
\hline $\mathbf{T}_{\mathbf{4}}$ & Amaranthus packed in 300 gauge polyethylene bag with 1 per cent perforation and stored under ZECC. \\
\hline $\mathbf{T}_{\mathbf{5}}$ & Amaranthus packed in plastic crates and stored under ZECC. \\
\hline $\mathbf{T}_{\mathbf{6}}$ & Amaranthus packed in 200 gauge polyethylene bag without perforation and stored under ambient condition \\
\hline $\mathbf{T}_{\mathbf{7}}$ & Amaranthus packed in 200 gauge polyethylene bag with 1 per cent perforation and stored under ambient condition \\
\hline $\mathbf{T}_{\mathbf{8}}$ & Amaranthus packed in 300 gauge polyethylene bag without perforation and stored under ambient condition \\
\hline $\mathbf{T}_{\mathbf{9}}$ & Amaranthus packed in 300 gauge polyethylene bag with 1 per cent perforation and stored under ambient condition \\
\hline $\mathbf{T}_{\mathbf{1 0}}$ & Amaranthus packed in plastic crates and stored under ambient condition. \\
\hline
\end{tabular}


Table.1c Effect of different packaging materials and storage temperature on physiological loss in weight (per cent) of amaranthus - 52 to 72 has $\left(3^{\text {rd }}\right.$ day after storage $)$

\begin{tabular}{|c|c|c|c|c|c|c|}
\hline \multirow[t]{2}{*}{ Treatments } & \multicolumn{6}{|c|}{$2^{\text {nd }}$ Day after storage (DAS) } \\
\hline & 52 HAS & 56 HAS & 60 HAS & 64 HAS & 68 HAS & 72 HAS \\
\hline $\mathbf{T}_{1}$ & 1.10 & 1.20 & 1.30 & 1.70 & 1.80 & 2.00 \\
\hline $\mathbf{T}_{2}$ & 1.20 & 1.30 & 1.50 & 1.80 & 1.90 & 2.50 \\
\hline $\mathbf{T}_{3}$ & 1.30 & 1.40 & 1.60 & 2.00 & 2.20 & 2.60 \\
\hline $\mathbf{T}_{4}$ & 2.10 & 2.10 & 2.30 & 2.50 & 2.50 & 3.00 \\
\hline $\mathbf{T}_{5}$ & 17.50 & 19.00 & 20.50 & 22.00 & 26.50 & 27.50 \\
\hline $\mathbf{T}_{6}$ & 1.20 & 1.40 & 1.60 & 2.00 & 2.00 & 2.50 \\
\hline $\mathbf{T}_{7}$ & 2.00 & 2.00 & 2.20 & 2.40 & 2.50 & 3.00 \\
\hline $\mathbf{T}_{8}$ & 2.10 & 2.30 & 2.50 & 3.00 & 3.00 & 3.50 \\
\hline $\mathbf{T}_{9}$ & 3.20 & 3.30 & 3.50 & 3.60 & 3.70 & 4.00 \\
\hline $\mathbf{T}_{10}$ & 45.50 & 47.50 & 48.00 & 49.50 & 52.50 & 55.00 \\
\hline SEd & 2.7599 & 2.8962 & 2.9574 & 3.0734 & 3.3336 & 3.4122 \\
\hline CD (0.05) & $5.7570 * *$ & $6.0414 * *$ & $6.1690 * *$ & $6.4111 * *$ & $6.9538 * *$ & $7.2846 * *$ \\
\hline
\end{tabular}

Treatment details

\begin{tabular}{|c|c|}
\hline $\mathbf{T}_{\mathbf{1}}$ & Amaranthus packed in $\mathbf{2 0 0}$ gauge polyethylene bag without perforation and stored under ZECC \\
\hline $\mathbf{T}_{\mathbf{2}}$ & Amaranthus packed in 200 gauge polyethylene bag with 1 per cent perforation and stored under ZECC. \\
\hline $\mathbf{T}_{\mathbf{3}}$ & Amaranthus packed in 300 gauge polyethylene bag without perforation and stored under ZECC. \\
\hline $\mathbf{T}_{\mathbf{4}}$ & Amaranthus packed in 300 gauge polyethylene bag with 1 per cent perforation and stored under ZECC. \\
\hline $\mathbf{T}_{\mathbf{5}}$ & Amaranthus packed in plastic crates and stored under ZECC. \\
\hline $\mathbf{T}_{\mathbf{6}}$ & Amaranthus packed in 200 gauge polyethylene bag without perforation and stored under ambient condition \\
\hline $\mathbf{T}_{\mathbf{7}}$ & Amaranthus packed in 200 gauge polyethylene bag with 1 per cent perforation and stored under ambient \\
& condition \\
\hline $\mathbf{T}_{\mathbf{8}}$ & Amaranthus packed in 300 gauge polyethylene bag without perforation and stored under ambient condition \\
\hline $\mathbf{T}_{\mathbf{9}}$ & Amaranthus packed in 300 gauge polyethylene bag with 1 per cent perforation and stored under ambient \\
\hline $\mathbf{T}_{\mathbf{1 0}}$ & Amaranthus packed in plastic crates and stored under ambient condition. \\
\hline
\end{tabular}


Table.1d Effect of different packaging materials and storage temperature on physiological loss in weight (per cent) of amaranthus - 76 to 96 has $\left(4^{\text {th }}\right.$ day after storage $)$

\begin{tabular}{|c|c|c|c|c|c|c|}
\hline \multirow[t]{2}{*}{ Treatments } & \multicolumn{6}{|c|}{$2^{\text {nd }}$ Day after storage (DAS) } \\
\hline & 76 HAS & 80 HAS & 84 HAS & 88 HAS & 92 HAS & 96 HAS \\
\hline $\mathbf{T}_{1}$ & 2.30 & 2.50 & 2.80 & 3.00 & 3.50 & 4.00 \\
\hline $\mathbf{T}_{2}$ & 2.70 & 2.80 & 3.00 & 3.50 & 4.00 & 4.50 \\
\hline $\mathbf{T}_{3}$ & 3.00 & 3.00 & 3.20 & 3.70 & 4.50 & 4.90 \\
\hline $\mathbf{T}_{4}$ & 3.30 & 3.60 & 3.70 & 4.20 & 4.70 & 5.20 \\
\hline $\mathbf{T}_{5}$ & 28.50 & 29.50 & 31.50 & 32.50 & 35.00 & 37.00 \\
\hline $\mathbf{T}_{6}$ & 2.80 & 2.90 & 3.00 & 3.60 & 4.20 & 4.70 \\
\hline $\mathbf{T}_{7}$ & 3.10 & 3.50 & 3.50 & 4.00 & 4.50 & 5.00 \\
\hline $\mathbf{T}_{8}$ & 3.80 & 3.90 & 4.00 & 4.50 & 5.00 & 5.50 \\
\hline $\mathbf{T}_{9}$ & 4.20 & 4.50 & 4.70 & 5.00 & 5.50 & 6.00 \\
\hline $\mathbf{T}_{10}$ & 57.00 & 59.00 & 61.00 & 62.00 & 64.00 & 66.00 \\
\hline SEd & 3.6222 & 3.7511 & 3.9041 & 3.9899 & 4.1675 & 4.3318 \\
\hline CD (0.05) & $7.5558 * * *$ & $7.8247 * *$ & $8.1439 * * *$ & $8.3227 * *$ & $8.6933 * *$ & $9.0360 * *$ \\
\hline
\end{tabular}

Treatment details

\begin{tabular}{|c|c|}
\hline $\mathbf{T}_{\mathbf{1}}$ & Amaranthus packed in 200 gauge polyethylene bag without perforation and stored under ZECC \\
\hline $\mathbf{T}_{\mathbf{2}}$ & Amaranthus packed in 200 gauge polyethylene bag with 1 per cent perforation and stored under ZECC. \\
\hline $\mathbf{T}_{\mathbf{3}}$ & Amaranthus packed in 300 gauge polyethylene bag without perforation and stored under ZECC. \\
\hline $\mathbf{T}_{\mathbf{4}}$ & Amaranthus packed in 300 gauge polyethylene bag with 1 per cent perforation and stored under ZECC. \\
\hline $\mathbf{T}_{\mathbf{5}}$ & Amaranthus packed in plastic crates and stored under ZECC. \\
\hline $\mathbf{T}_{\mathbf{6}}$ & Amaranthus packed in 200 gauge polyethylene bag without perforation and stored under ambient condition \\
\hline $\mathbf{T}_{\mathbf{7}}$ & Amaranthus packed in 200 gauge polyethylene bag with 1 per cent perforation and stored under ambient condition \\
\hline $\mathbf{T}_{\mathbf{8}}$ & Amaranthus packed in 300 gauge polyethylene bag without perforation and stored under ambient condition \\
\hline $\mathbf{T}_{\mathbf{9}}$ & Amaranthus packed in 300 gauge polyethylene bag with 1 per cent perforation and stored under ambient condition \\
\hline $\mathbf{T}_{\mathbf{1 0}}$ & Amaranthus packed in plastic crates and stored under ambient condition. \\
\hline
\end{tabular}


Table.1e Effect of different packaging materials and storage temperature on physiological loss in weight (per cent) of amaranthus - 24, 48, 72 and 96 has $\left(1^{\text {st }}, 2^{\text {nd }}, 3^{\text {rd }}\right.$ and $4^{\text {th }}$ day after storage $)$

\begin{tabular}{|c|c|c|c|c|}
\hline & 1DAS & 2DAS & 3 DAS & 4 DAS \\
\hline Treatments & 24 HAS & 48 HAS & 72 HAS & 96 HAS \\
\hline $\mathbf{T}_{\mathbf{1}}$ & 0.20 & 1.00 & 2.00 & 4.00 \\
\hline $\mathbf{T}_{\mathbf{2}}$ & 0.40 & 1.10 & 2.50 & 4.50 \\
\hline $\mathbf{T}_{\mathbf{3}}$ & 0.80 & 1.20 & 2.60 & 4.90 \\
\hline $\mathbf{T}_{\mathbf{4}}$ & 1.50 & 2.10 & 3.00 & 5.20 \\
\hline $\mathbf{T}_{\mathbf{5}}$ & 9.00 & 16.00 & 27.50 & 37.00 \\
\hline $\mathbf{T}_{\mathbf{6}}$ & 0.40 & 1.10 & 2.50 & 4.70 \\
\hline $\mathbf{T}_{\mathbf{7}}$ & 1.40 & 1.90 & 3.00 & 5.00 \\
\hline $\mathbf{T}_{\mathbf{8}}$ & 1.70 & 2.10 & 3.50 & 5.50 \\
\hline $\mathbf{T}_{\mathbf{9}}$ & 2.20 & 3.20 & 4.00 & 6.00 \\
\hline $\mathbf{T}_{\mathbf{1 0}}$ & 25.00 & 43.00 & 55.00 & 66.00 \\
\hline $\mathbf{S E d}$ & $\mathbf{1 . 5 0 8 9}$ & $\mathbf{2 . 5 9 8 7}$ & $\mathbf{3 . 4 1 2 2}$ & $\mathbf{4 . 3 3 1 8}$ \\
\hline $\mathbf{C D}(\mathbf{0 . 0 5})$ & $\mathbf{3 . 1 4 7 4} * *$ & $\mathbf{5 . 4 2 0 8} * *$ & $\mathbf{7 . 2 8 4 6} * *$ & $\mathbf{9 . 0 3 6 0} * *$ \\
\hline
\end{tabular}

Treatment details

\begin{tabular}{|c|c|}
\hline $\mathbf{T}_{1}$ & $\begin{array}{l}\text { Amaranthus packed in } 200 \text { gauge polyethylene bag without perforation } \\
\text { and stored under ZECC }\end{array}$ \\
\hline $\mathbf{T}_{2}$ & $\begin{array}{c}\text { Amaranthus packed in } 200 \text { gauge polyethylene bag with } 1 \text { per cent perforation } \\
\text { and stored under ZECC. }\end{array}$ \\
\hline $\mathbf{T}_{3}$ & $\begin{array}{l}\text { Amaranthus packed in } 300 \text { gauge polyethylene bag without perforation and } \\
\text { stored under ZECC. }\end{array}$ \\
\hline $\mathbf{T}_{4}$ & $\begin{array}{c}\text { Amaranthus packed in } 300 \text { gauge polyethylene bag with } 1 \text { per cent perforation } \\
\text { and stored under ZECC. }\end{array}$ \\
\hline $\mathbf{T}_{5}$ & Amaranthus packed in plastic crates and stored under ZECC. \\
\hline $\mathbf{T}_{6}$ & $\begin{array}{l}\text { Amaranthus packed in } 200 \text { gauge polyethylene bag without perforation and } \\
\text { stored under ambient condition }\end{array}$ \\
\hline $\mathbf{T}_{7}$ & $\begin{array}{c}\text { Amaranthus packed in } 200 \text { gauge polyethylene bag with } 1 \text { per cent perforation } \\
\text { and stored under ambient condition }\end{array}$ \\
\hline $\mathbf{T}_{8}$ & $\begin{array}{c}\text { Amaranthus packed in } 300 \text { gauge polyethylene bag without perforation and } \\
\text { stored under ambient condition }\end{array}$ \\
\hline $\mathbf{T}_{9}$ & $\begin{array}{c}\text { Amaranthus packed in } 300 \text { gauge polyethylene bag with } 1 \text { per cent perforation } \\
\text { and stored under ambient condition }\end{array}$ \\
\hline $\mathbf{T}_{10}$ & Amaranthus packed in plastic crates and stored under ambient condition. \\
\hline
\end{tabular}

However, the differences among the treatment were found to the wide from 72 HAS ( $3^{\text {rd }}$ day) onwards to 96 HAS ( $4^{\text {th }}$ day).

Two different five kinds of treatments were studied 200, 300 gauge without and with perforation and plastic crates and stored under ZECC. Among the different treatments, amaranthus packed in 200 gauge polyethylene bag without perforation and stored under ZECC $\left(T_{1}\right)$ was found to best as it is recorded in the lowest physiological loss in weight. It 
was followed by the second-best treatment $\mathrm{T}_{2}$ (Amaranthus packed in 200 gauge polyethylene bag with 1 percent perforation and stored under ZECC). The highest PLW was recorded in amaranthus packed in plastic crates and stored under ZECC $\left(\mathrm{T}_{5}\right)$.

Another five different kinds of treatments were studied 200, 300 gauge without and with perforation and plastic crates and stored under ambient condition. Among the different treatments, the treatment $\left(\mathrm{T}_{6}\right)$ amaranthus packed in 200 gauge polyethylene bag without perforation and stored under ambient condition was found to best as it is recorded in the lowest physiological loss in weight. It was followed by the second-best treatment $\mathrm{T}_{7}$ (Amaranthus packed in 200 gauge polyethylene bag with 1 percent perforation and stored under ambient condition). The highest PLW was recorded in amaranthus packed in plastic crates and stored under ambient condition $\left(\mathrm{T}_{10}\right)$.

Overall, among the 10 treatments, amaranthus packed in 200 gauge polyethylene bag without vent and stored under ZECC $\left(\mathrm{T}_{1}\right)$ recorded the lowest physiological loss in weight compared to other treatments. It was followed by $\left(T_{2}\right)$ amaranthus packed in 200 gauge polyethylene bag with 1 per cent perforation and stored under ZECC. ZECC storage whether packed or not, maintained freshness, in addition to increasing the shelf life. It is evident that the rate of physiological and biochemical changes in leaves during storage were dependent on temperature and relative humidity. Hence, the slow rate of these changes in leaves in ZECC might be due to low temperature and high relative humidity maintained in the ZECC (Srinivasa and Reddy, 1996). Under low temperature and high relative humidity in the ZECC, the rate of respiration and other enzymatic activities were probably at a slow rate thereby delaying the senescence process in the leaves
(Roy and Khurdiya,1986). From this, it could be informed that higher humidity and low temperature in ZECC were significantly effective in keeping down the physiological weight loss due to which it slowed down the metabolic activities like respiration and transpiration. Whereas at ambient temperature has a higher temperature and low relative humidity results in rapid transpiration and respiration. Reduced weight loss in ZECC storage has been reported by Waskar et al., (1999) for bottle gourd.

Among the various packaging materials used, amaranthus packed in 200 gauge polyethylene bags without perforation was found to best in both ambient and ZECC.

Compared to different perforation of 200 and 300 gauge polyethylene bag without and with perforation was used, amaranthus packed in 200 gauge polyethylene bag without perforation was found to best in both ambient and ZECC.

The role of polyethylene in reducing the PLW has been earliest reported by Jindal et al., (2005) and this might be due to restriction of diffusion of gases resulting in a slow rate of respiration and moisture loss. Among the different storage methods, amaranthus stored inside the ZECC is found to be better than ambient conditions irrespective of packaging materials. Either in ambient condition or ZECC, polyethylene packaging reduced PLW compared to plastic crates which may be due to the modified atmosphere created in the package which in turn might have slowed down the moisture loss ultimately reduced physiological loss in weight (Miller and Risse, 1998). Further, the results observed in the present study were in conformity with Sadaswari et al., (1972) in oranges, Dostal (1977), Siambhi (1982) in the carrot reported that wrapping carrots in polyethylene bags reduced the weight loss during storage. 
References

Dosal, H.C. (1997). Pre-cooling harvested vegetables, American vegetable grower, 25 (8): 12-13

Jindal. S., Beniwal, L.S., Godara, N.R. and R.P Sihag, (2005), Studies on the Shelf life of Sapota fruits with polyethylene packaging. Haryana Journal of Horticultural Sciences, 34: 253-255.

Miller, M.R. and Risse, L.A. (1998). Recent research of film wrapping of fresh produce in Florida, Balaban publisher, $1521-1530$

Roy, S.K. and Khurdiya, D.S. (1986). Studies on evaporative cooled zero energy input cool chamber for the storage of horticultural produce. Indian Food Packer, 40 (6): 26 - 31

Saimbhi, M.S. (1982). Shelf life of carrot varieties as influenced by prepackaging in polyethylene bags.
Punjab Vegetable Grower, 17 (18): 48-50.

Sankaran, M. 1999. Effect of pre-packing and storage temperatures on the quality and shelf life of fresh bitter gourd fruits. (Momordica charantia L) M.Sc. (Hort.) Thesis. Horticultural College and Research Institute, Periyakulam.

Srinivasa, V and Reddy, T.V. (1996). Extension of post-harvest life of palak by packing and cool chamber storage, Journal of Maharashtra Agricultural Universities, 21(1): 33-35

Waskar, D.P., B. Yadav and V.K.Garande. (1999). Influence of various packaging materials on storage behaviour of bottle gourd under different storage conditions. Indian Journal of Agricultural Research, 33(44): 287292.

\section{How to cite this article:}

Marichamy, M. S., J. Jyothsna, S. Harini, V. Bhuvaneswari Devi, B. Rajapriya, A. Rajalakshmi and Ahaljith, R. 2020. Studies on Effect of Different Packaging Materials and Storage Temperature on Physiological Loss in Weight of Amaranthus (Amaranthus viridis). Int.J.Curr.Microbiol.App.Sci. 9(10): 720-731. doi: https://doi.org/10.20546/ijcmas.2020.910.087 